CAE Working Paper \#05-15

Capital Gains: Blue Machines and Red Machines

by

Luís Aguiar-Conraria

and

Karl Shell

August 2005 


\title{
Capital Gains: Blue Machines and Red Machines*
}

\author{
Luís Aguiar-Conraria \\ Cornell University and NIPE - Universidade do Minho \\ Karl Shell \\ Cornell University
}

August 15, 2005

\begin{abstract}
Capital gains play an essential role in the intertemporal allocation of resources, but they can also fuel self-fulfilling bubbles. The simple case of 2 "identical" capitals is analyzed in an OG model. The only trajectory in which expectations are realized at every date is the one in which blue machines and red machines have the same price. If ever their prices differ, then there is a "bubble" which must burst in finite time.
\end{abstract}

Key Words: bubbles, capital gains, heterogeneous capital, irreversible investment, overlapping generations, Tobin's q

\section{Introduction}

Capital gains (and losses) are essential to the workings of private-ownership economies. Changes in asset prices signal anticipated changes in relative scarcities. Capital gains can, however, fuel self-perpetuating bubbles, some of which will eventually burst.

\footnotetext{
*Paper prepared for the Singapore Economic Review conference, August 4-6, 2005.
} 
For individuals (or corporations or universities), the capital gains on (growth) stocks are no less income than the interest paid on bonds or the dividends paid on (value) stocks. For individuals, the increase in wealth resulting from realized or unrealized capital gains is saving just as are payroll deductions deposited in a 401K retirement account.

The analysis of capital gains requires (1) more than one asset in a macrodynamic model and (2) careful attention to the workings of the asset market. We take our cue from the old paper by Shell and Stiglitz (1967) ${ }^{1}$. In Shell and Stiglitz, there are two capitals, perfect foresight, but savings behavior is not based on individual utility maximization. In the present paper, we analyze a two-capital production technology very much like that in Shell and Stiglitz (1967). We replace the ad hoc consumption function of Shell-Stiglitz with utility-maximizing individuals in the overlapping-generations model of Diamond (1965) extended to allow for two capitals. We also assume that capital, once installed, cannot be directly consumed. ${ }^{2}$ Therefore, investments are irreversible allowing for the prices of used machines to fall below their reproduction costs, i.e. for a Tobin's $q$ which is less than 1 .

We will in a subsequent paper study the more general 2-capital technology of Shell and Stiglitz, but here we focus on the very special (but revealing) case in which the machines are perfect substitutes as factors of production and have identical replacement costs if newly produced. Hence we refer to the two capitals as, respectively, blue machines and red machines. However, we allow used machinery to have market prices that depend on the machine color (blue or red). Capitals are non-malleable: the color of a machine cannot be altered. This assumption allows for the possibility of different prices between blue and red machines. We adopt discrete time and assume that individuals live for two periods.

If the prices of blue and red machines are always equal, the model re-

\footnotetext{
${ }^{1}$ Also see, e.g., Shell, Sidrauski, and Stiglitz (1969), Caton and Shell (1971), Burmeister, Caton, Dobell, and Ross (1973), Shell (1972), and Burmeister and Graham (1974).

${ }^{2}$ See Magill and Quinzii (2003).
} 
duces in essence to the Diamond model adjusted to incorporate irreversible investment:

- In the steady state there is a unique overall capital to labor ratio, which is stable.

- If the overall capital to labor ratio is sufficiently high, then the price of used machinery is below its reproduction cost and there is no new investment. Otherwise the prices of used machines equal their reproduction costs. Once the economy enters the range in which investment is positive, it does not return to the no-investment regime.

If, however, the price of blue machines is allowed to differ from that of red machines, we have:

- The price of the lower-priced machine will become zero or negative in finite time, revealing that this path is not a long-run perfect foresight competitive equilibrium trajectory.

- On the unique competitive equilibrium trajectory in which expectations are always fulfilled, the price of red machines equals the price of blue machines at every date.

\section{The Model}

In each period, there is a generation of identical, old consumers and a generation of identical, young consumers. Each young consumer inelastically supplies one unit of labor. The old do not work. The labor force $L$ grows at the rate $n \geq 0$, so we have

$$
L_{t+1}=(1+n) L_{t},
$$


where $L_{t}$ is the number of consumers born in year $t=0,1, \ldots$. Consumers have identical utility functions

$$
u\left(x_{t}^{y}, x_{t}^{o}\right)=\log x_{t}^{y}+\beta \log x_{t}^{o}
$$

where $x_{t}^{y}$ is consumption when young and $x_{t}^{o}$ is consumption when old.

Production is given by the 1-sector, 3-output, 3-input model:

$$
C_{t}+Z_{t}^{1}+Z_{t}^{2}=Y_{t}=\left(K_{t}^{1}+K_{t}^{2}\right)^{\alpha} L_{t}^{1-\alpha}
$$

where $\alpha$ is a scalar between 0 and $1, K_{t}^{1}>0$ and $K_{t}^{2}>0$ are, respectively, the quantities of blue machines and red machines, $Y_{t}>0$ is undifferentiated output, $C_{t} \geq 0$ is consumption, $Z_{t}^{1} \geq 0$ and $Z_{t}^{2} \geq 0$ are respectively gross investment in blue machines and red machines, all at time $t$. Investment is irreversible and capital goods are non-malleable (i.e. blue machines cannot be turned into red ones, nor vice versa) since $Z_{t}^{i} \geq 0$. Let $\mu>0$ be the rate of depreciation on each type of machinery:

$$
K_{t+1}^{i}=(1-\mu) K_{t}^{i}+Z_{t}^{i}
$$

for $i=1,2$. Denote by lower case letters quantities normalized by $L$, e.g., $k_{t}=K_{t} / L_{t}$, so we have

$$
c_{t}+z_{t}^{1}+z_{t}^{2}=y_{t}=\left(k_{t}^{1}+k_{t}^{2}\right)^{\alpha}
$$

and

$$
(1+n) k_{t+1}^{i}=(1-\mu) k_{t}^{i}+z_{t}^{i}
$$

for $i=1,2$. Under competition, factors are rewarded by their marginal products, so we have

$$
r_{t}^{i}=\alpha\left(k_{t}^{1}+k_{t}^{2}\right)^{\alpha-1}>0
$$

for $i=1,2$, and

$$
w_{t}=\left(k_{t}^{1}+k_{t}^{2}\right)^{\alpha}-\alpha\left(k_{t}^{1}+k_{t}^{2}\right)^{\alpha}>0,
$$


where $r_{t}^{i}$ is the rental rate on type- $i$ capital and $w_{t}$ is the wage rate. Of course, blue machines and red machines yield the same marginal product,

$$
r_{t}^{1}=r_{t}^{2}=\alpha\left(k_{t}^{1}+k_{t}^{2}\right)^{\alpha-1}=r_{t} .
$$

We assume that individuals possess perfect foresight about price changes. Hence equilibrium in the used machinery market requires that the rate of return (including capital gains) on blue machines be equal to the rate of return on red machines, or

$$
\frac{(1-\mu) p_{t+1}^{1}+r_{t+1}^{1}}{p_{t}^{1}}=\frac{(1-\mu) p_{t+1}^{2}+r_{t+1}^{2}}{p_{t}^{2}}=\rho_{t+1},
$$

where $p^{i} \geq 0$ is the current price of machine $i$ in terms of the consumption good. The common rate of return is denoted by $\rho$. Equations 8 and 9 yield

$$
\frac{(1-\mu) p_{t+1}^{1}+r_{t+1}}{p_{t}^{1}}=\frac{(1-\mu) p_{t+1}^{2}+r_{t+1}}{p_{t}^{2}} .
$$

The consumer chooses $\left(x_{t}^{y}, x_{t}^{o}\right)$ and savings $s_{t} \geq 0$ to maximize

$$
u\left(x_{t}^{y}, x_{t}^{o}\right)=\log x_{t}^{y}+\beta \log x_{t}^{o}
$$

subject to

$$
x_{t}^{y}=w_{t}-s_{t}
$$

and

$$
x_{t}^{o}=\rho_{t+1} s_{t},
$$

where $0<\beta<1$ is the discount factor, "log" denotes the natural logarithm, and $s_{t}$ is savings. The consumer's problem can be stated more succinctly:

$$
\max _{s_{t}} \log \left(w_{t}-s_{t}\right)+\beta \log \left(\rho_{t+1} s_{t}\right),
$$

where $0 \leq s_{t} \leq w_{t}$. The solution to this problem is given by:

$$
s_{t}=\frac{\beta}{1+\beta} w_{t} .
$$




\section{Competitive Equilibrium}

Young consumers use their savings to buy capital that they will rent in period $t$ and sell in period $t+1$. In competitive equilibrium, the supply of machinery must equal savings, or

$$
(1+n)\left(p_{t}^{1} k_{t+1}^{1}+p_{t}^{2} k_{t+1}^{2}\right)=\frac{\beta}{1+\beta} w_{t}=\frac{\beta}{1+\beta}(1-\alpha)\left(k_{t}^{1}+k_{t}^{2}\right)^{\alpha} .
$$

Consumption per head is always positive, so we can normalize prices by the price of current consumption. Under competition, firms will only produce goods with the highest market price. Hence we have

$$
\max \left(p_{t}^{1}, p_{t}^{2}\right) \leq 1
$$

If $\max \left(p_{t}^{1}, p_{t}^{2}\right)<1$, then $z_{t}^{1}=z_{t}^{2}=0$. If $\max \left(p_{t}^{1}, p_{t}^{2}\right)=1$, then the machine with the lower price will not be produced. If $p_{t}^{1}=p_{t}^{2}=1$, then the composition of investment is indeterminate. Define $z$ by

$$
z_{t}=z_{t}^{1}+z_{t}^{2}
$$

and $\sigma$ by

$$
\sigma_{t}=z_{t}^{1} / z_{t}
$$

We have

$$
\sigma_{t}\left\{\begin{array}{clll}
=1 & \text { if } p_{t}^{1}>p_{t}^{2} & \text { and } & z_{t}>0 \\
\in[0,1] & \text { if } p_{t}^{1}=p_{t}^{2} & \text { and } & z_{t}>0 \\
=0 & \text { if } p_{t}^{1}<p_{t}^{2} & \text { and } & z_{t}>0 \\
\text { undefined } & \text { if } & & \\
& & & z_{t}=0
\end{array} .\right.
$$

Definition 1 Given initial per capita capital stocks $\left(k_{0}^{1}, k_{0}^{2}\right)$, a long-run perfect-foresight competitive equilibrium is given by the sequence of allocations $\left\{k_{t+1}^{1}, k_{t+1}^{2}, s_{t}, x_{t}^{y}, x_{t}^{o}\right\}_{t=0}^{\infty}$ and the sequence of non-negative prices $\left\{r_{t}^{1}, r_{t}^{2}, p_{t}^{1}, p_{t}^{2}\right\}_{t=0}^{\infty}$ such that equations (7), (8) and (12), and the market-clearing conditions 10 and 13 are satisfied. 


\section{Steady State}

Since $r^{1}=r^{2}=r$, we have from $(10)$ that $p^{1}=p^{2}$. Unless $\max \left(p_{t}^{1}, p_{t}^{2}\right)=1$, $\left(k_{t}^{1}+k_{t}^{2}\right)$ must fall by $(5)$. Hence we have

$$
p^{1}=p^{2}=1
$$

From (4) and (13), we derive the overall steady-state capital stock per head, $k^{1}+k^{2}$, and output per head, $y$, in the steady state:

$$
\begin{aligned}
k^{1}+k^{2} & =\left(\frac{\beta}{(1+\beta)} \frac{(1-\alpha)}{(1+n)}\right)^{\frac{1}{1-\alpha}} \\
y & =\left(k^{1}+k^{2}\right)^{\alpha}
\end{aligned}
$$

We see that $\left(k^{1}+k^{2}\right)$ and $y$ are uniquely determined and positive but that $k^{1}$ and $k^{2}$ are indeterminate. Summarizing we have:

Proposition 1 In the steady state, the overall capital to labor ratio $\left(k^{1}+k^{2}\right)$ and output per worker $y$ are uniquely determined, but $\sigma=k^{1} /\left(k^{1}+k^{2}\right)$ is any number in $[0,1]$.

\section{Existence and uniqueness of the competi- tive equilibrium trajectory}

Given initial endowments, we will show that there is a competitive equilibrium path, which is unique in $\left\{c_{t}, y_{t}, k_{t}^{1}+k_{t}^{2}, p_{t}^{1}, p_{t}^{2}\right\}_{0}^{\infty}$. First it is useful to show that if gross investment is ever positive it will remain positive.

Proposition 2 If gross investment is strictly positive at time $t$, it will also be strictly positive at time $t+1$

Proof. If $z_{t}>0$ then $\max \left(p_{t}^{1}, p_{t}^{2}\right)=1$. Without loss of generality assume that $p_{t}^{1}=1$. If $p_{t}^{2}=1$ the proof is trivial so consider $p_{t}^{2}<1$. Given this we 
have $(1-\mu) k_{t}^{1}+p_{t}^{2}(1-\mu) k_{t}^{2}<\frac{\beta}{1+\beta}(1-\alpha)\left(k_{t}^{1}+k_{t}^{2}\right)^{\alpha}$ and we want to show that

$$
p_{t+1}^{1}(1-\mu) k_{t+1}^{1}+p_{t+1}^{2}(1-\mu) k_{t+1}^{2}<\frac{\beta}{1+\beta}(1-\alpha)\left(k_{t+1}^{1}+k_{t+1}^{2}\right)^{\alpha}
$$

First note that the no-arbitrage condition 10 immediately implies that since $p_{t}^{1}>p_{t}^{2}$ we must have $\frac{p_{t+1}^{1}}{p_{t}^{1}}>\frac{p_{t+1}^{2}}{p_{t}^{2}}$, which implies that $p_{t+1}^{1}>p_{t+1}^{2}$. So it is enough to show that

$$
(1-\mu) k_{t+1}^{1}+p_{t+1}^{2}(1-\mu) k_{t+1}^{2}<\frac{\beta}{1+\beta}(1-\alpha)\left(k_{t+1}^{1}+k_{t+1}^{2}\right)^{\alpha} .
$$

But if $p_{1, t+1}=1$ we must have $p_{2, t+1}<p_{2, t}$, so we only need to show that

$$
(1-\mu) k_{t+1}^{1}+p_{t}^{2}(1-\mu) k_{t+1}^{2}<\frac{\beta}{1+\beta}(1-\alpha)\left(k_{t+1}^{1}+k_{t+1}^{2}\right)^{\alpha} .
$$

Using $k_{t+1}^{2}=\frac{(1-\mu)}{(1+n)} k_{t}^{2}$ we have

$$
(1-\mu)\left(k_{t+1}^{1}+p_{t}^{2} \frac{(1-\mu)}{(1+n)} k_{t}^{2}\right)<\frac{\beta}{1+\beta}(1-\alpha)\left(k_{t+1}^{1}+\frac{(1-\mu)}{(1+n)} k_{t}^{2}\right)^{\alpha} .
$$

After some rearranging and using $k_{t+1}^{1}+p_{t}^{2}(1-\mu) k_{t}^{2}=\frac{\beta}{(1+\beta)} \frac{(1-\alpha)}{(1+n)}\left(k_{t}^{1}+k_{t}^{2}\right)^{\alpha}$, and $p_{t}^{2}(1-\mu) k_{t}^{2} \frac{n}{1+n}>0$ it is immediate that it is enough to show that

$$
\frac{(1-\mu)}{(1+n)}\left(k_{t}^{1}+k_{t}^{2}\right)^{\alpha}<\left(\frac{\beta}{(1+\beta)} \frac{(1-\alpha)}{(1+n)}\left(k_{t}^{1}+k_{t}^{2}\right)^{\alpha}+(1-\mu)\left(1-p_{t}^{2}\right) k_{t}^{2}-n \frac{(1-\mu)}{(1+n)} k_{t}^{2}\right)^{\alpha}
$$

which is equivalent to

$$
\left(\frac{1-\mu}{1+n}\right)^{\frac{1}{\alpha}} k_{t}^{1}+p_{t}^{2}(1-\mu) k_{t}^{2}+\left(\frac{1-\mu}{1+n}\right)^{\frac{1}{\alpha}} k_{t}^{2}<\frac{\beta}{(1+\beta)} \frac{(1-\alpha)}{(1+n)}\left(k_{t}^{1}+k_{t}^{2}\right)^{\alpha}+\frac{1-\mu}{1+n} k_{t}^{2},
$$

which is true because

$$
\left(\frac{1-\mu}{1+n}\right)^{\frac{1}{\alpha}} k_{t}^{1}+p_{t}^{2}(1-\mu) k_{t}^{2}<\frac{\beta}{1+\beta} \frac{1-\alpha}{1+n}\left(k_{t}^{1}+k_{t}^{2}\right)^{\alpha}
$$

and

$$
\left(\frac{1-\mu}{1+n}\right)^{\frac{1}{\alpha}} k_{t}^{2}<\frac{1-\mu}{1+n} k_{t}^{2}
$$


Proposition 3 The sequence of prices $\left\{p_{t}^{1}, p_{t}^{2}\right\}_{t=0}^{\infty}$ such that $p_{t}^{1}=p_{t}^{2}$ and the market clearing condition (13) are satisfied is compatible with a competitive equilibrium.

Proof. Case 1. Suppose that

$$
(1-\mu) k_{t}^{1}+(1-\mu) k_{t}^{2}<\frac{\beta}{1+\beta}(1-\alpha)\left(k_{t}^{1}+k_{t}^{2}\right)^{\alpha},
$$

then new investment is positive and we have $p_{t}^{1}=p_{t}^{2}=1$. The arbitrage condition is automatically satisfied and the dynamics reduce to the dynamics of a standard Diamond model with

$$
\left(k_{t+1}^{1}+k_{t+1}^{2}\right)=\frac{\beta}{(1+\beta)} \frac{(1-\alpha)}{(1+n)}\left(k_{t}^{1}+k_{t}^{2}\right)^{\alpha} .
$$

Case 2. Now suppose we have

$$
(1-\mu) k_{t}^{1}+(1-\mu) k_{t}^{2} \geq \frac{\beta}{1+\beta}(1-\alpha)\left(k_{t}^{1}+k_{t}^{2}\right)^{\alpha} .
$$

In this case investment cannot be strictly positive and prices should adjust so that

$$
p_{t}^{1}(1-\mu) k_{t}^{1}+p_{t}^{2}(1-\mu) k_{t}^{2}=\frac{\beta}{1+\beta}(1-\alpha)\left(k_{t}^{1}+k_{t}^{2}\right)^{\alpha},
$$

with $p_{t}^{1}=p_{t}^{2} \leq 1$. In period 1 , we will have $k_{t+1}^{i}=\frac{(1-\mu)}{(1+n)} k_{t}^{i}, i=1,2$, and $\left(k_{t+1}^{1}+k_{t+1}^{2}\right)^{\alpha}=\left(\frac{1-\mu}{1+n}\right)^{\alpha}\left(k_{t}^{1}+k_{t}^{2}\right)^{\alpha}$. So it is a matter of time for the inequality to flip, and the economy moves to case 1 , where it will stay by the previous proposition.

Proposition 4 The equilibrium described in proposition 3 is unique.

Proof. We prove by contradiction. Without loss of generality assume that $p_{t}^{1}>p_{t}^{2}$. The arbitrage condition tells us that $(1-\mu) \frac{p_{t+1}^{1}}{p_{t}^{1}}+\frac{r_{t+1}}{p_{1, t}}=$ $(1-\mu) \frac{p_{t+1}^{2}}{p_{t}^{2}}+\frac{r_{t+1}}{p_{t}^{2}}$. Since $p_{t}^{1}>p_{t}^{2}$ we must have $\frac{p_{t+1}^{1}}{p_{t}^{1}}>\frac{p_{t+1}^{2}}{p_{t}^{2}}$. Since $p_{t}^{1}>p_{t}^{2}$, we 
must have $p_{t+1}^{1}>p_{t+1}^{2}$, and then $p_{t+2}^{1}>p_{t+2}^{2}$, and so on. Therefore, if any new investment is made, it will be in capital 1 (blue machines).

Case 1. Suppose that

$$
p_{t}^{1}(1-\mu) k_{t}^{1}+p_{t}^{2}(1-\mu) k_{t}^{2}<\frac{\beta}{1+\beta}(1-\alpha)\left(k_{t}^{1}+k_{t}^{2}\right)^{\alpha} .
$$

This implies that there is new investment in capital 1, and hence:

$$
\begin{aligned}
p_{t}^{1} k_{t+1}^{1} & =\frac{\beta}{(1+\beta)} \frac{(1-\alpha)}{(1+n)}\left(k_{t}^{1}+k_{t}^{2}\right)^{\alpha}-p_{t}^{2}(1-\mu) k_{t}^{2} \\
k_{t+1}^{2} & =\frac{(1-\mu)}{(1+n)} k_{t}^{2}
\end{aligned}
$$

and

$$
p_{t}^{1}=1
$$

Case 2. Suppose we have

$$
p_{t}^{1}(1-\mu) k_{t}^{1}+p_{t}^{2}(1-\mu) k_{t}^{2}=\frac{\beta}{1+\beta}(1-\alpha)\left(k_{t}^{1}+k_{t}^{2}\right)^{\alpha} .
$$

There is no new investment, $p_{t}^{1} \leq 1$ and $k_{t+1}^{i}=\frac{(1-\mu)}{(1+n)} k_{t}^{i}, i=1,2$. It is a matter of time to move to Case 1, where the economy will stay. So we can focus our attention in Case 1.

To rule out the possibility an equilibrium, we only need to show that in finite time $p^{2}$ will become negative. Since $p_{t}^{1}=1$ for all $t$, the arbitrage condition tells us that $p_{t+1}^{2}-p_{t}^{2}=\frac{r_{t+1}}{1-\mu}\left(p_{t}^{2}-1\right)$. Note that the right hand side is negative and bounded away from zero. So in finite time $p_{2}$ will become negative. 


\section{Computed Examples - how long before the bubble bursts?}

Our numerical exercises are inspired in part by Atkinson $(1969)^{3}$. The parameter values used in our computations are given in Table 1.

\begin{tabular}{ccccccc}
\multicolumn{5}{c}{ Table 1: } & Assumed Parameter Values \\
\hline \hline$\alpha$ & $\beta$ & $\mu$ & $n$ & $k_{0}^{1}$ & $k_{0}^{2}$ \\
0.4 & 0.6 & 0.55 & 0 & 1 & 5
\end{tabular}

In the 2-period-lifetime OG model we identify "youth" with the working years and "old age" with the retirement years. Therefore, one period in the OG model corresponds to roughly 20 years. So $\beta=0.6$ corresponds to an annual discount factor on the order of $97.5 \%$, while $\mu=0.55$ corresponds to an annual depreciation rate of about $4 \%$.

In Table 2 we display an efficient, bubble-free growth path. The initial overall capital to labor ratio, $k_{0}^{1}+k_{0}^{2}$, is large, so the initial gross investments are zero and initial prices are bellow unity: $p_{0}^{1}=p_{0}^{2}=0.170639376$. The total value of capital converges to its equilibrium steady-state value:, $k^{1}+k^{2}=$ 0.083234658. There are no bubbles: $p_{t}^{1}=p_{t}^{2}$ for $t=0,1,2, \ldots$

In Figure 1, we present the evolution of $\left(k_{t}^{1}+k_{t}^{2}\right)$. After 6 periods, or about 120 years, the steady state is nearly achieved. In Figure $2, p_{t}^{1}=p_{t}^{2}$ is plotted. In period zero, prices of used machines are less than 1 and only in period 4 do they become 1. As we can see in Figure 3, there is no gross investment as long as prices are smaller than 1. We plot the trajectories of savings $s_{t}$ (curve marked with squares) and gross investment $z_{t}$ (curve marked circles). $s_{t}$ declines with time, as total capital decreases. $z_{t}$ increases sharply in periods 4 and 5 and and then quickly approaches its steady state value.

\footnotetext{
${ }^{3}$ See especially pages $144-148$.
} 
Table 2: Bubble-free growth path

\begin{tabular}{ccccc}
\hline \hline$t$ & $k_{t}^{1}+k_{t}^{2}$ & $p_{t}^{1}=p_{t}^{2}$ & $s_{t}$ & $z_{t}$ \\
0 & 6 & 0.170639376 & 0.460726 & 0 \\
1 & 2.7 & 0.275519122 & 0.334756 & 0 \\
2 & 1.215 & 0.444860901 & 0.243228 & 0 \\
3 & 0.54675 & 0.718284888 & 0.176725 & 0 \\
4 & 0.246038 & 1.000 & 0.128405 & 0.017688 \\
5 & 0.110717 & 1.000 & 0.093297 & 0.043474 \\
6 & 0.093297 & 1.000 & 0.087122 & 0.045139 \\
7 & 0.087122 & 1.000 & 0.084768 & 0.045563 \\
8 & 0.084768 & 1.000 & 0.083845 & 0.045699 \\
9 & 0.083845 & 1.000 & 0.083478 & 0.045748 \\
10 & 0.083478 & 1.000 & 0.083332 & 0.045767 \\
11 & 0.083332 & 1.000 & 0.083274 & 0.045774 \\
12 & 0.083274 & 1.000 & 0.083250 & 0.045777 \\
13 & 0.083250 & 1.000 & 0.083241 & 0.045778 \\
14 & 0.083241 & 1.000 & 0.083237 & 0.045778 \\
15 & 0.083237 & 1.000 & 0.083235 & 0.045779 \\
16 & 0.083236 & 1.000 & 0.083235 & 0.045779 \\
17 & 0.083235 & 1.000 & 0.083235 & 0.045779 \\
18 & 0.083235 & 1.000 & 0.083235 & 0.045779
\end{tabular}

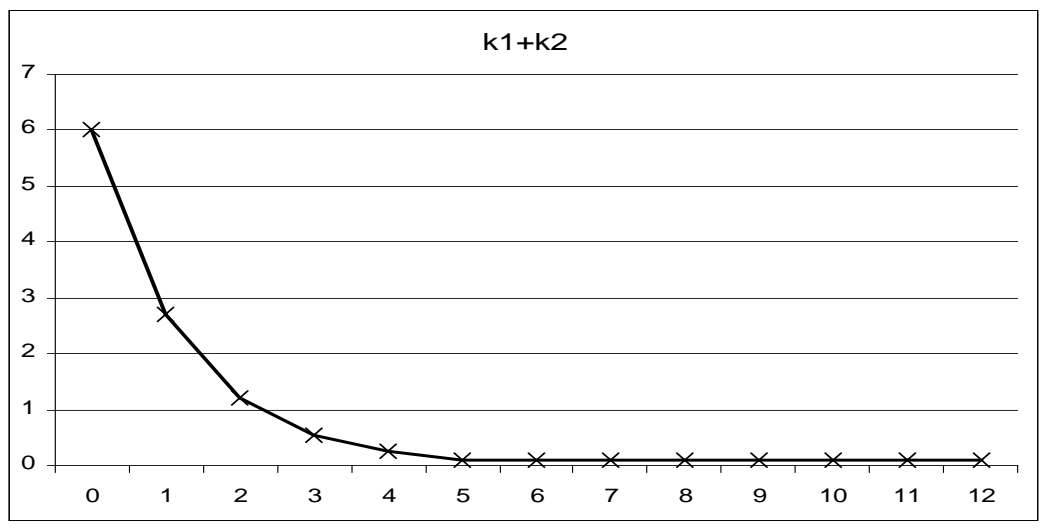

Figure 1: Evolution of the overall capital/labor ratio on the bubble-free path 


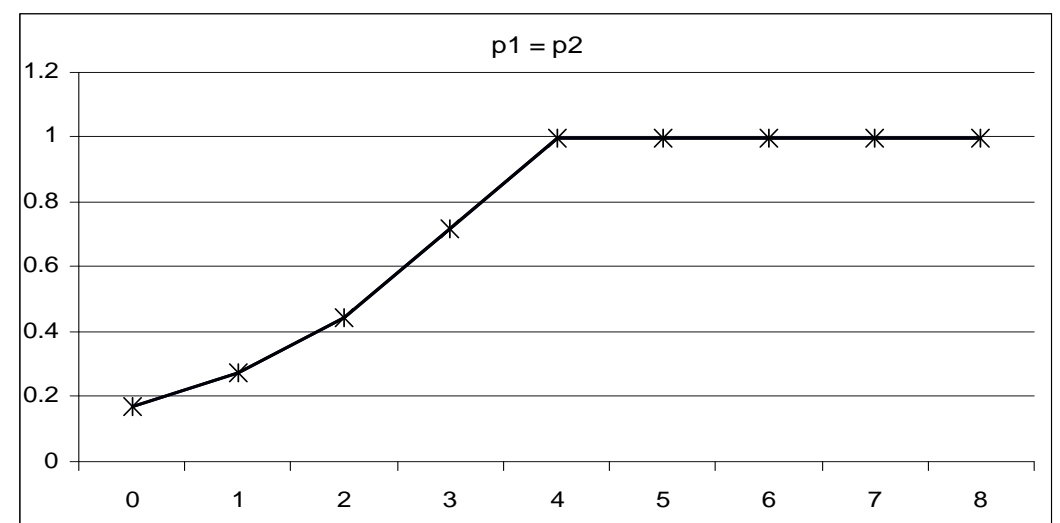

Figure 2: Evolution of prices on the bubble-free path

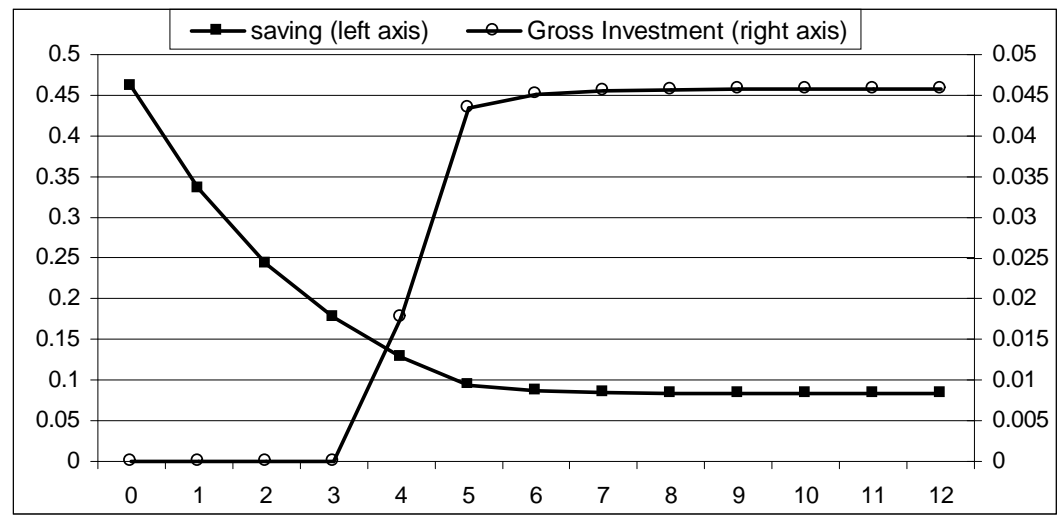

Figure 3: Evolution of savings and gross investment on the bubble-free path

For Table 3, we perturb the previous exercise. Instead of setting $p_{0}^{1}=p_{0}^{2}$, we allow for $p_{0}^{2}$ to be slightly larger than $p_{0}^{1}$. That is we introduce a positive red-machine "bubble".

In Figure 4, the $p_{t}^{1}$ path is marked by circles, and the $p_{t}^{2}$ path is marked by squares. Since $p_{0}^{1}$ is smaller than $p_{0}^{2}$, the yield for the different machines is different with

$$
\frac{r}{p_{0}^{1}}>\frac{r}{p_{0}^{2}}
$$


Table 3: Red-machine bubble growth path

\begin{tabular}{ccccccc}
\hline \hline$t$ & $k_{t}^{1}$ & $k_{t}^{2}$ & $p_{t}^{1}$ & $p_{t}^{2}$ & $s_{t}$ & $z_{t}$ \\
0 & 1 & 5 & 0.170639000 & 0.170639451 & 0.460726 & 0 \\
1 & 0.45 & 2.25 & 0.275517436 & 0.275519459 & 0.334756 & 0 \\
2 & 0.2025 & 1.0125 & 0.444853339 & 0.444862414 & 0.243228 & 0 \\
3 & 0.091125 & 0.455625 & 0.718250971 & 0.718291671 & 0.176725 & 0 \\
4 & 0.041006 & 0.205031 & 0.999826513 & 1.000 & 0.128405 & 0.017692 \\
5 & 0.018453 & 0.109956 & 0.999298119 & 1.000 & 0.098997 & 0.041219 \\
6 & 0.008304 & 0.090699 & 0.996799366 & 1.000 & 0.089216 & 0.044676 \\
7 & 0.003737 & 0.085491 & 0.98467151 & 1.000 & 0.085582 & 0.045455 \\
8 & 0.001682 & 0.083926 & 0.925127357 & 1.000 & 0.084176 & 0.045709 \\
9 & 0.000757 & 0.083476 & 0.631441715 & 1.000 & 0.083632 & 0.045853 \\
10 & 0.000341 & 0.083417 & $<0$ & 1.000 & 0.083444 &
\end{tabular}

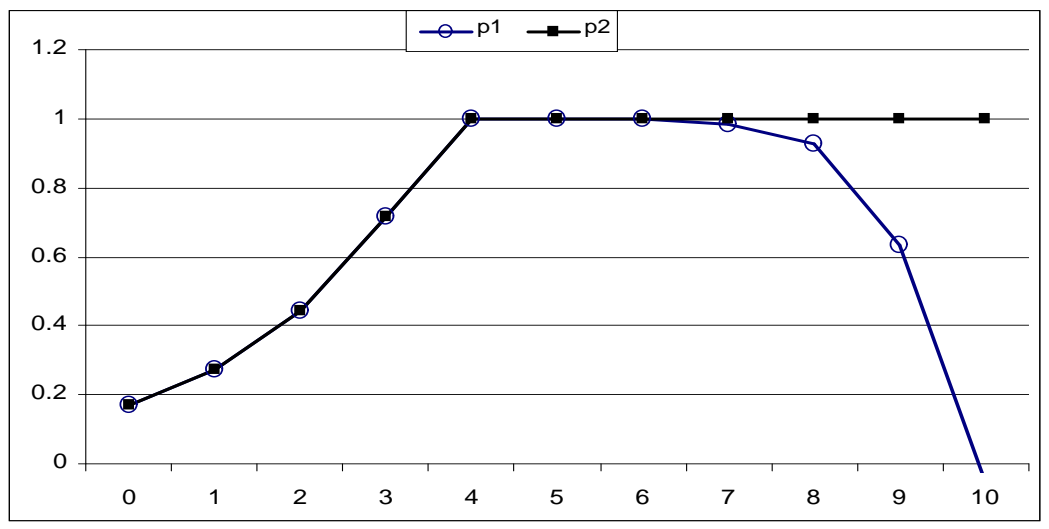

Figure 4: Bubble prices

So, for the asset market to clear, the capital gains on red machines must be greater than the capital gains on blue machines. The bubble on red machines must burst before period 10 .

If the initial bubble is greater, then the bubble will burst more quickly. For example, if $p_{0}^{1}=0.16$ and $p_{0}^{2}=0.172767251$ the bubble must burst by period 3 .

We have investigated economies with parameter values different from those given in Table 1 . We have replaced $\mu=0.55$ with $\mu=0.9$, corresponding to a yearly depreciation rate of $10 \%$. The initial prices consistent 
with bubble-free competitive equilibrium are $p_{0}^{1}=p_{0}^{2}=0.767877191$. In period 1 , we have $p_{1}^{1}=p_{1}^{2}=1.00$ After 5 periods, $\left(k_{t}^{1}+k_{t}^{2}\right)$ becomes close to its steady state value $\left(k^{1}+k^{2}\right)=0.08323$. If we have a small bubble on red machines, given by $p_{0}^{1}=0.767877$ and $p_{0}^{2}=0.767877230$, then by period 6 $p_{t}^{1}$ becomes negative. Hence the red machine bubble must burst by period 6 .

It is not surprising that the higher is the depreciation rate the quicker the bubble will burst. The larger are the depreciation rates, other things equal, the smaller are the capital gains. Hence changes in the prices will have to be even bigger to compensate for the differences in the yields on the machines, leading to shorter lived bubbles. If the depreciation rate is $100 \%$, there would be no capital gains and there would be no room for perfect-foresight bubbles.

\section{Concluding Remarks}

Blue machines are technologically identical to red machines. The central planner and the representative infinitely-lived agent are indifferent to the color of machinery. The shadow price of a blue machine is equal to the shadow price of a red machine on the optimal trajectory.

We investigate prices and capital gains (and losses) on blue machines and red machines in a simple OG model. On the unique competitive path in which expectations are always fulfilled, the market price of blue machines is always equal to the market price of red machines. If there are complete futures markets in machinery, then this bubble-free path is the only one that will be pursued. But we are talking here about a large (potentially infinite) number of futures markets.

In the real world, futures markets extend out to only a few years at most. In our computed examples, bubble-trajectories will eventually be revealed to be disequilibrium paths, but only after decades or more. The question re- 
mains: Does the ownership economy steer away from bubbles that eventually burst? If so, how is this accomplished? If not, what are the consequences for public policy?

\section{Biographical Note}

Our particular model has important antecedents: (1) The technology is essentially that of Shell-Stiglitz $(1967)^{4}$ except that the present paper employs discrete time and explicitly allows for irreversible capital so that Tobin's $q$ can be less than unity. ${ }^{5}$ Except for the introduction of 2 capitals and the irreversibility of investment, we are using the OG model of Diamond $(1965)^{6}$.

\section{References}

[1] Atkinson, A. B. (1969), The timescale of economic models: How long is the long run?, The Review of Economic Studies, 36, 137-152.

[2] Burmeister, E., Caton, C., Dobell, A. and Ross, S. (1973), The "Saddlepoint Property" and the Structure of Dynamic Heterogeneous Capital Good Models, Econometrica, 41, 79-95.

[3] Burmeister, E. and Graham, D. (1974), Multi-sector Economic Models with Continuous Adaptive Expectations, Review of Economic Studies, vol. 41, 323-36.

[4] Caton, C. and Shell, K. (1971), An Exercise in the Theory of Heterogeneous Capital Accumulation, The Review of Economic Studies, Vol. 37, $13-22$.

\footnotetext{
${ }^{4}$ See page 594 .

${ }^{5}$ See Magill and Quinzii (2003).

${ }^{6}$ Capital gains and losses do not arise in the pure Diamond model.
} 
[5] Diamond, P., 1965, National Debt in a Neoclassical Growth Model, American Economic Review, 55, 1126-1150.

[6] Magill, M. and Quinzii, M. (2003), Nonshiftable Capital, Affine Price Expectations and Convergence to the Golden Rule, Journal of Mathematical Economics 39, 239-272

[7] Shell, K. (1972), On Competitive Dynamical Systems, in Differential Games and Related Topics (H.W. Kuhn and G.P. Szegö, eds.), Amsterdam: North-Holland , 449-476.

[8] Shell, K., Sidrauski, M. and Stiglitz, J. (1967), Capital Gains, Income, and Saving, The Review of Economic Studies, vol. 36, 15-26.

[9] Shell, K. and Stiglitz, J. (1967), The Allocation of Investment in a Dynamic Economy, Quarterly Journal of Economics, vol. 81, 592-609. 